\title{
SISTEMA PARA LA GESTIÓN DE
} INFORMACIÓN DE LOS SERVICIOS Y MEDIOS DE CÓMPUTO EN EL GRUPO EMPRESARIAL CONSTRUCCIONES GRANMA

\section{MANAGEMENT INFORMATION SYSTEM OF THE SERVICES AND COMPUTER RESOURCES IN THE GRUPO EMPRESARIAL CONSTRUCCIONES GRANMA}

\author{
Yudi Castro Blanco ${ }^{1}$ \\ Susana Teresa Carbonell Hernández ${ }^{2}$ \\ Eric Ismael Leonard Brizuela ${ }^{3}$
}

1. Máster en Informática aplicada. Profesora Asistente de la Universidad de Granma, Bayamo, Cuba. E-Mail: ycastrob@udg.co.cu

2. Ingeniero Informático. Especialista en el Grupo Empresarial Construcciones Granma, Bayamo, Cuba. E-mail: susana@dir.gecgr.co.cu

3. Ingeniero Informático. Profesor Asistente de la Universidad de Granma, Bayamo, Cuba. E-Mail: eleonardb@udg.co.cu

Castro Blanco, Y., Carbonell Hernández, S.T. y Leonard Brizuela, E.I. (2016). App vs papel como soporte para el usuario. Efectos de las Tics en la adherencia a un tratamiento de fisioterapia. 3C TIC: Cuadernos de desarrollo aplicados a las TIC, 5(3), 65-76. DOI: <http://dx.doi.org/10.17993/3ctic.2016.53.65-76/>. 


\section{RESUMEN}

En el Grupo Empresarial Construcciones Granma, en Cuba, se lleva a cabo el registro del gran volumen de información relacionada con los servicios y medios de cómputo de los que se dispone en el grupo empresarial y en las siete empresas subordinadas. Entre los elementos que se controlan se encuentran las computadoras, sus periféricos, componentes internos y características técnicas, además, los cambios o reemplazos, mantenimientos que se realizan y aspectos de la seguridad informática como la actualización de los programas y las auditorias. Su procesamiento se realiza de forma manual, invirtiéndose mucho tiempo debido a que se requiere la consulta de diferentes documentos, lo que provoca retrasos en la entrega de informes de carácter nacional. Atendiendo a estas necesidades, la presente investigación tiene como objetivo desarrollar una aplicación web. Para su implementación, se empleó el framework Django en la programación de páginas dinámicas, Phyton como lenguaje del lado del servidor, HTML5 como lenguaje del lado del cliente, con soporte de base de datos en SQLite y la metodología de desarrollo Extreme Programing. Con su empleo se facilita el registro y procesamiento de la información de manera rápida, permitiendo una mayor consistencia, seguridad y elevando el nivel de eficiencia desde el punto de vista organizativo, de control y analítico.

\section{ABSTRACT}

In the Grupo Empresarial Construcciones Granma (GREMCO), is carried out the registration of a large information volume related to the services and computing resources which is available in the business group and at the seven subordinate companies. Among the elements that are controlled are computers, peripherals, internal components and technical characteristics, besides, the changes or replacements, maintenances that are accomplished and aspects of security informatics like the update of the programs and the auditing. Its processing is done manually, inverting a long time because it require to consult different documents provoking delays in information processing and the delivery of reports. Attending to this needs, this research aims to develop a web application. For its implementation, the Django framework was used in the programming of dynamic pages, Phyton like the language of the server side, HTML5 like the language in the customer side and with data base support SQLite and the methodology Extreme Programming. With its use is facilitate the recording and processing information, enabling greater consistency, security and raising the level of efficiency from the organizational point of view, control and analytical.

\section{PALABRAS CLAVE}

Aplicación web; gestión de servicio y medios de cómputo; gestión de información.

\section{KEY WORDS}

Web Application; service management and computer resources; Information Management. 


\section{INTRODUCCIÓN}

Desde que el hombre comenzó a comunicarse, vio la necesidad de recopilar y procesar información que con el tiempo se han ido perfeccionado gracias a los avances de la ciencia y la técnica, unidos al surgimiento de la computadora. Por eso, considera Aladro (2011) que los países desarrollados basan su crecimiento en la aplicación y la programación estratégica de las herramientas computacionales y han definido políticas que los inducirán a su permanencia en el dinamismo mundial de los próximos años. De este modo, la presencia de la computación en los sectores productivos es un factor determinante para su funcionamiento.

Nada ni nadie escapan al paradigma y la nueva sociedad que ha creado las telecomunicaciones, la informática e Internet, y el mundo de la construcción no es ninguna excepción. Según Ferrada y Serpell (2009), cada vez son más las empresas constructoras que se involucran en gestionar sus principales procesos de diseño, construcción e ingeniería empleando las tecnologías de la información y la comunicación para cumplir con calidad su encargo social.

En Cuba, el Ministerio de la Construcción es el organismo rector encargado de dirigir, ejecutar en lo que le compete y controlar la política del estado y el gobierno en cuanto a los servicios de diseño, ingeniería y construcción, producción de materiales de construcción y del sistema de la vivienda. Cumple un importante rol en el desarrollo del país, tanto cultural como económico, pues a través de la construcción se satisfacen las necesidades de infraestructura de la mayoría de las actividades económicas y sociales de la nación (MICONS, 2014).

El Grupo Empresarial Construcciones Granma (GREMCO) es una de las dependencias del Ministerio de la Construcción. El mismo cuenta con una alta productividad y eficiencia económica logrando construcciones competitivas, dotado de un personal motivado y elevado sentido de pertenencia. También cuenta con toda la industria modernizada y máxima calidad en las obras minimizando los daños en el entorno con una fuerte imagen corporativa que permite satisfacer a los clientes nacionales e internacionales.

La Dirección Técnica de GREMCO tiene como principal función gestionar toda la información referente a los servicios y medios de cómputo de los que se dispone en el grupo empresarial y en las siete empresas subordinadas. Entre ellas, se encuentran las computadoras, sus periféricos, componentes internos y sus características técnicas. Además se registran los cambios o remplazos y mantenimientos que se realizan $\mathrm{y}$ aspectos de la seguridad informática, como la actualización y autorización de los programas y las auditorias, incluyendo los servicios que se prestan. Todo el control que lleva a cabo constituye un criterio de medida a nivel nacional, lo que requiere la entrega de informes de forma periódica. Han sido detectadas algunas deficiencias en este proceso: 
- Se invierte mucho tiempo para su realización, pues se requiere la consulta de varios documentos, lo que implica que se puedan cometer errores.

- Los documentos son vulnerables a pérdida, sustracción y deterioro.

- La información es enviada por correo electrónico a la Dirección Nacional de Informática y Comunicaciones del Ministerio de la Construcción, y hasta tanto no llegue no puede consultarse la situación actual de la misma.

- Para conocer el estado de los equipos es necesario consultar informes aislados provocando demoras en el proceso.

- Se requiere de gran cantidad de papeles y no existe seguridad en los documentos pudiendo existir alteración por personal no autorizado.

- No se desarrollan comparaciones entre empresas para un mismo indicador por período, que muestre la situación actual de los componentes y equipos de forma general.

No se halló ninguna propuesta informática que realice una gestión detallada de los servicios y medios de cómputo. Se encontró la propuesta de un modelo para la gestión automatizada e integrada de controles de seguridad informática (Montesino, Baluja y Porvén, 2013) y una metodología para su implementación (Miranda, Valdés, Pérez, Portelles y Sánchez, 2016). Estos estudios permiten profundizar sobre una parte del proceso que se gestionada en GREMCO, los procederes en la seguridad informática, permitiendo realizarse un reevaluar de la manera en la que se realiza según los estándar internacional.

Según Vidal y Araña (2012) y Torres (2015) la gestión de la información es el proceso de organizar la información, evaluar, presentar, comparar los datos en un determinado contexto, controlar la calidad, veracidad, que sea oportuna, significativa, exacta, útil y que esté disponible en el momento que se le necesite. Teniendo en cuenta esta consideración y las deficiencias detectadas en GREMCO, se consideran necesarias nuevas formas de gestión, o sea, mecanismos más eficaces de almacenamiento y procesamiento de los datos.

En aras de solucionar la ineficiencia en el proceso de gestión de la información relacionada con los servicios y medios de cómputo en GREMCO, se propone en esta investigación desarrollar una aplicación web que lo realice de manera rápida y eficiencia.

\section{METODOLOGÍA}

Inicialmente, se hizo un estudio en GREMCO para constatar las deficiencias en la gestión de la información relacionada con los servicios y medios de cómputo y determinar la mejor vía para solucionarlo. Se utilizaron métodos y técnicas como el análisis y síntesis para recopilar y procesar la información necesaria, la revisión documental para conocer con claridad los datos que son de interés, la entrevista para obtener datos detallados sobre su procesamiento, y la observación para ver las funcionalidades del jefe de la dirección técnica. Se realizó una búsqueda de sistemas informáticos que pudieran emplearse para resolver las deficiencias 
detectadas, y al no encontrarse ninguno, se desarrolló una aplicación web con este objetivo que se ajustara a sus necesidades.

Para su desarrollo se hizo un estudio bibliográfico sobre las posibles herramientas, tecnologías y lenguajes a utilizarse (Gutiérrez, 2008; Hernández y Greguas, 2010) según sus prestaciones, tendencias actuales y novedades, con el fin de mantener la mayor integración posible y seleccionar las adecuadas. Se empleó el proceso de desarrollo para guiar la metodología Extreme Programing o como también se le conoce XP, ya que es una metodología ágil que se basa en la realimentación continua entre el cliente y el equipo de desarrollo, comunicación fluida entre todos los participantes y simplicidad en las soluciones (Díaz, 2009; Tinoco, Rosales y Salas, 2010; Escobar y Gualteros, 2013; Bagarotti, Meneses y Arias, 2013). Se implementó haciendo uso de los lenguajes de programación del lado del cliente HTML 5 (Gauchat, 2012) y del lado del servidor Python (Challenger, Díaz y Becerra, 2014), este último permite la creación de páginas web dinámicas y facilita la conexión a diferentes tipos de servidores de bases de datos. Para la maquetación se utilizó Bootstrap, pues permite crear interfaces web con CSS y Javascript (Lessin, 2013). Como gestor de base de datos se hizo uso de SQLite, ya que cuenta con un completo soporte para tablas e índices en un único archivo por base de datos, soporte transaccional, rapidez (dos veces más veloz que MySQL y PostgreSQL), escaso tamaño y su completa portabilidad. Se utilizó el framework Django por las facilidades para la creación de sitios web complejos y su compatibilidad con Python. Las fases que se siguieron teniendo en cuenta lo que la metodología XP establece fueron las siguientes:

1. Exploración. En ella se definió el alcance del proyecto, se especificaron las funcionalidades que se deseaban informatizar para satisfacer las necesidades del cliente mediante 32 historias de usuario, estimándose el tiempo de desarrollo de cada una, el cual no era superior a los tres días. Entre las definidas se encuentran: actualizar y visualizar datos de las computadoras, componentes, periféricos, incidencias de la seguridad informática, comportamiento de la conectividad (enlace conmutado y arrendado), mantenimientos, cambios de piezas, los programas actualizados por computadoras, los datos de los usuarios con correo y conexión nacional e internacional, reporte general de computadoras por empresa, entre otras.

2. Plan de entrega. Se realizó una estimación detallada del tiempo a emplearse en el desarrollo de las historias de usuario y para eso se dividieron en tres iteraciones. En el cronograma de entregas se estimó que la duración de la primera iteración fuera de cuatro semanas, la segunda y la tercera tres semanas cada una.

3. Iteraciones. Se planificó la etapa de implementación, definiéndose las tareas para desarrollar cada historia de usuario en términos de diseño y programación, se describió cada una y se estimó el tiempo de duración.

4. Producción. Se diseñó, codificó y probó la aplicación web. Tras ser creada la primera iteración, se trabajó en las nuevas.

- Diseño: para lograr un sistema robusto, reutilizable y basados en las prácticas de la metodología seleccionada, se trabajó en un diseño sencillo y evolutivo. La base 
de datos se diseñó con 14 tablas normalizadas hasta la Forma Normal de BoyceCodd mediante la herramienta Embarcadero ER/Studio.

- Codificación: se tuvo en cuenta la reutilización de código para agilizar la implementación. Se empleó el método de indentación, popular en Python. Se estableció un estándar de codificación para lograr uniformidad y facilitar la comprensión del código.

- Pruebas: se realizaron pruebas unitarias y de aceptación para evaluar la calidad de la aplicación web de forma pragmática y descubrir errores. Las unitarias o de caja blanca fueron desarrolladas por los programadores para verificar el código, es decir, los detalles procedimentales (la lógica del sistema). Las de aceptación también llamadas pruebas funcionales o de caja negra fueron supervisadas por el cliente basándose en los requerimientos tomados de las historias de usuario, se verificó el resultado esperado de una transacción determinada, y se llevaron a cabo sobre la interfaz. Estas pruebas permitieron encontrar errores como: funciones incorrectas, errores de interfaz, errores en estructuras de datos, errores de inicialización, errores ortográficos, la no actualización correcta de los datos de una computadora y que no notificaba la actualización de un mantenimiento. Todos los errores detectados fueron corregidos a tiempo, lográndose una aplicación web de mayor calidad.

5. Mantenimiento. Durante el desarrollo se tuvieron en cuenta las opiniones del cliente. En esta etapa se identificaron necesidades que propiciaron cambios en la aplicación web, lo que dio lugar a mantenimiento de tipo adaptativo, correctivo y perfectivo.

6. Muerte del Proyecto. Se generó la documentación final y al no requerirse más cambios en la arquitectura, al cliente no tener más historias de usuario que incluir y al estar satisfecho con el sistema, pues éste generó los beneficios esperados, se dio por terminada la aplicación web.

Una vez que finalizó la implementación de la aplicación web, esta se desplegó en GREMCO y se capacitó al personal que interactúa con la misma. Además, se proporcionó un manual de usuario que contiene detalles de cómo se opera con ella para que pudiera evacuar cualquier duda sobre su manejo en el futuro.

\section{RESULTADOS}

La aplicación web cuenta con una serie de funcionalidades que la hacen una herramienta útil para gestionar la información relacionada con los servicios y medios de cómputo en GREMCO y las siete empresas subordinadas. En su desarrollo se intentó satisfacer las necesidades del cliente. Su interfaz es cómoda y facilita la navegación. Es configurable, permitiendo ser implantado con gran facilidad en cualquier grupo empresarial o empresa que controle la misma información.

En aras de garantizar la seguridad y confiabilidad de los datos que se gestionan, cuenta con una jerarquía de usuario para acceder a cualquier opción del sistema, dígase: introducir, 


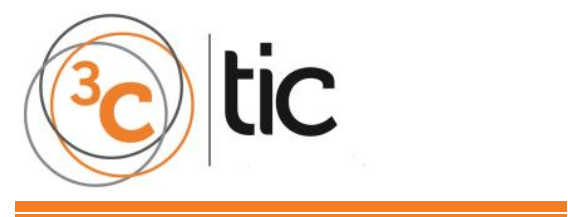

modificar o eliminar e incluso visualizar los reportes, garantizándose así que la información que se introduzca la hagan personas debidamente autorizadas. Se tuvo en cuenta que solo accedan a los registros de la empresa a la que pertenecen y que a nivel de grupo empresarial se tenga acceso a todo. La contraseña se encriptó en la base de datos mediante el método md5, que permite controlar las operaciones que se realizan mediante una especie de log que guarda los datos con la fecha y hora. La figura 1 muestra la página de inicio.

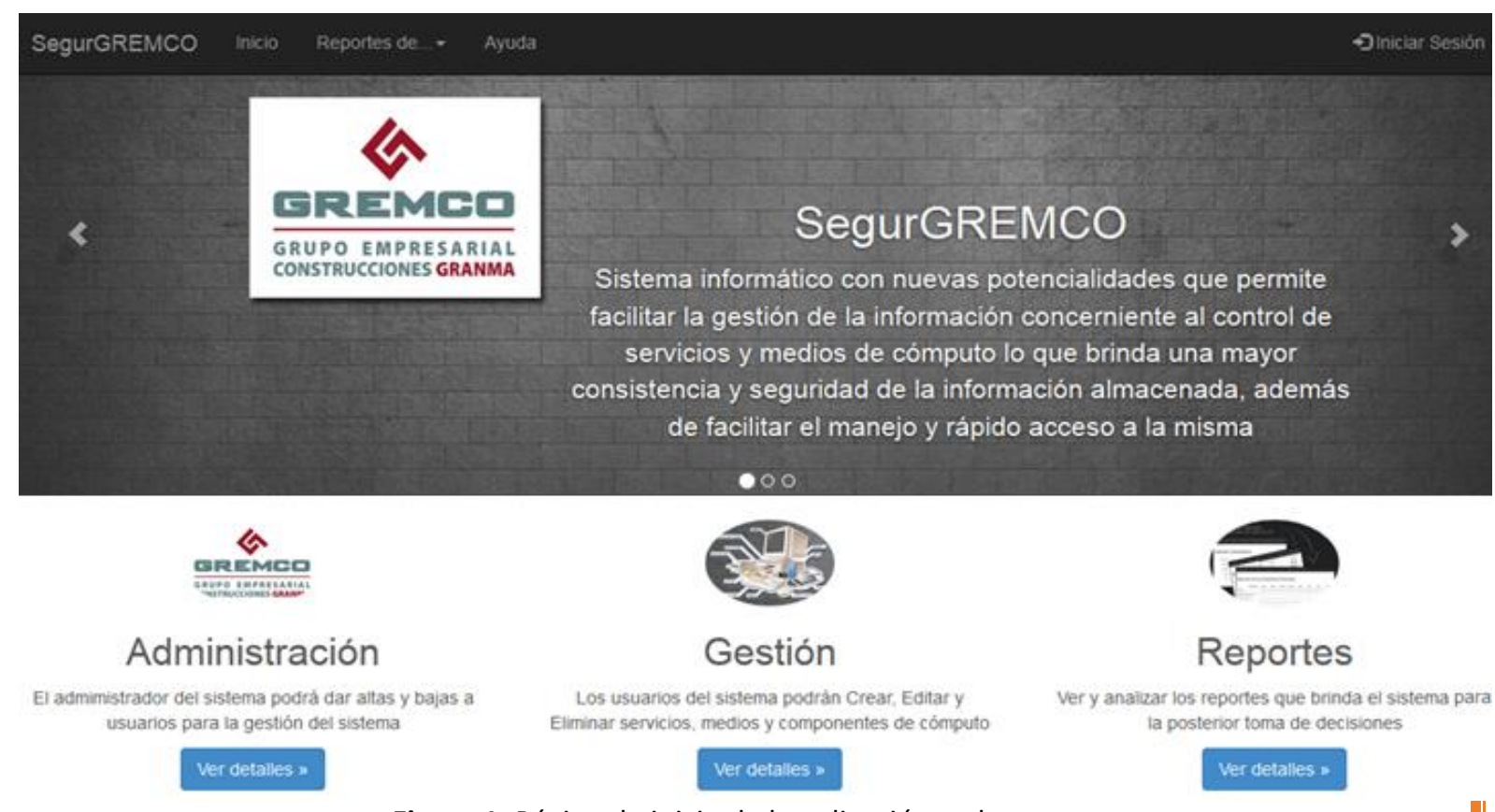

Figura 1. Página de inicio de la aplicación web.

Entre la información que se gestiona mediante la aplicación web se encuentra la referente a las computadoras, en la figura 2 se observa el formulario con esta opción. Se siguió el mismo diseño para que sea cómodo al usuario, por ejemplo, el botón aceptar siempre va a indicar almacenar la información en la base de datos. Se tuvieron en cuenta algunos elementos que ayudan a que los errores humanos se minimicen, como lo es en este caso, la selección en vez de entrarse del tipo de computadora y su estado, además que solo se pueda introducir en cada campo el tipo de dato correcto (letra, número o ambos). 

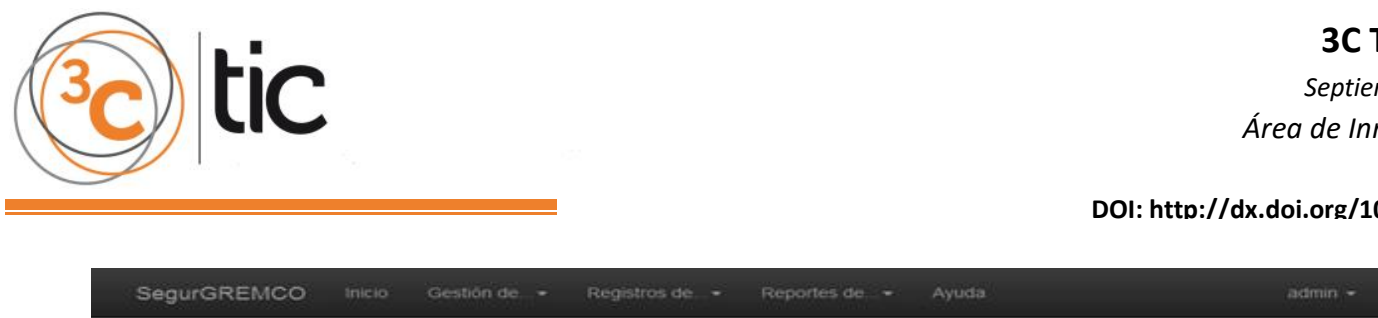

Gestionar computadoras

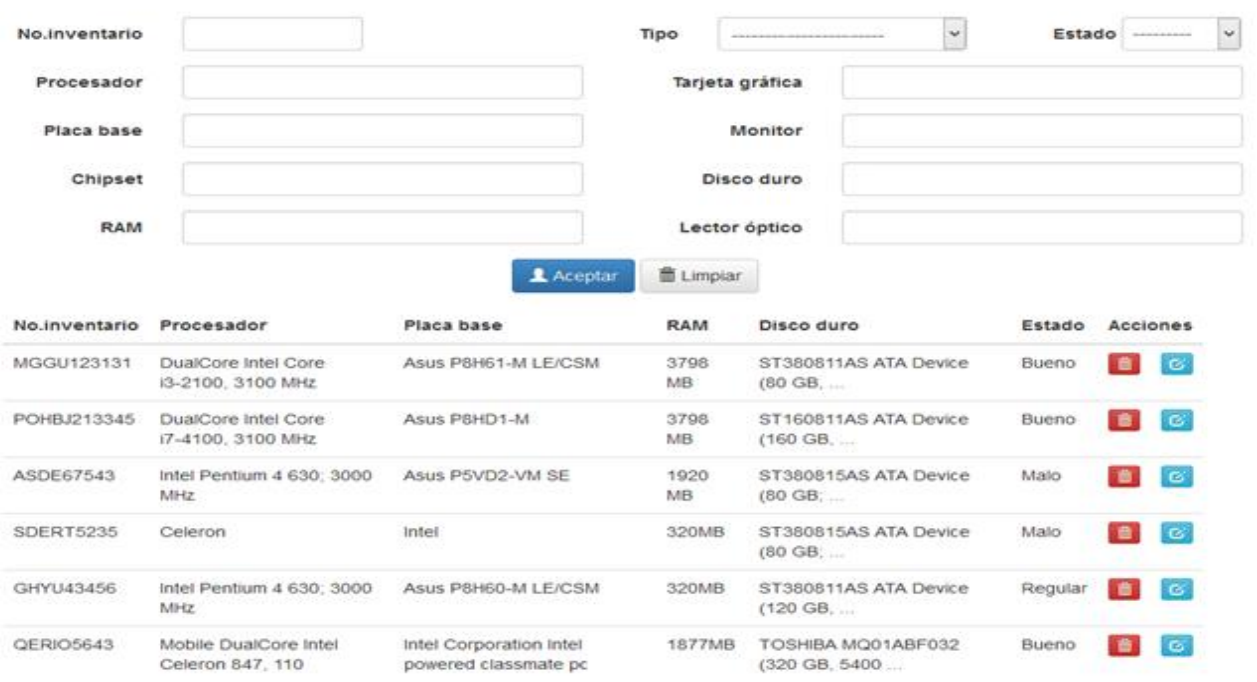

Figura 2. Gestión de los datos de las computadoras mediante la aplicación web.

También se pueden gestionar los periféricos, las incidencias de seguridad informática que se producen, el comportamiento de la conectividad en la empresa como la cantidad de enlace conmutado y arrendado, datos de los usuarios con correo y conexión nacional e internacional, el registro de mantenimiento y cambios de piezas que se realizan a las computadoras, entre otras informaciones.

Para obtenerse los reportes solo es necesario acceder a la opción y son emitidos de forma rápida según modelos oficiales, lo que sustituye los cálculos manuales que antes se hacían. También se pueden realizar comparaciones entre empresas e indicadores. Por ejemplo, como se muestra en la figura 3 , donde se indica la cantidad de periféricos de los que se dispone y su estado (malo (M), regular (R) y bien (B).

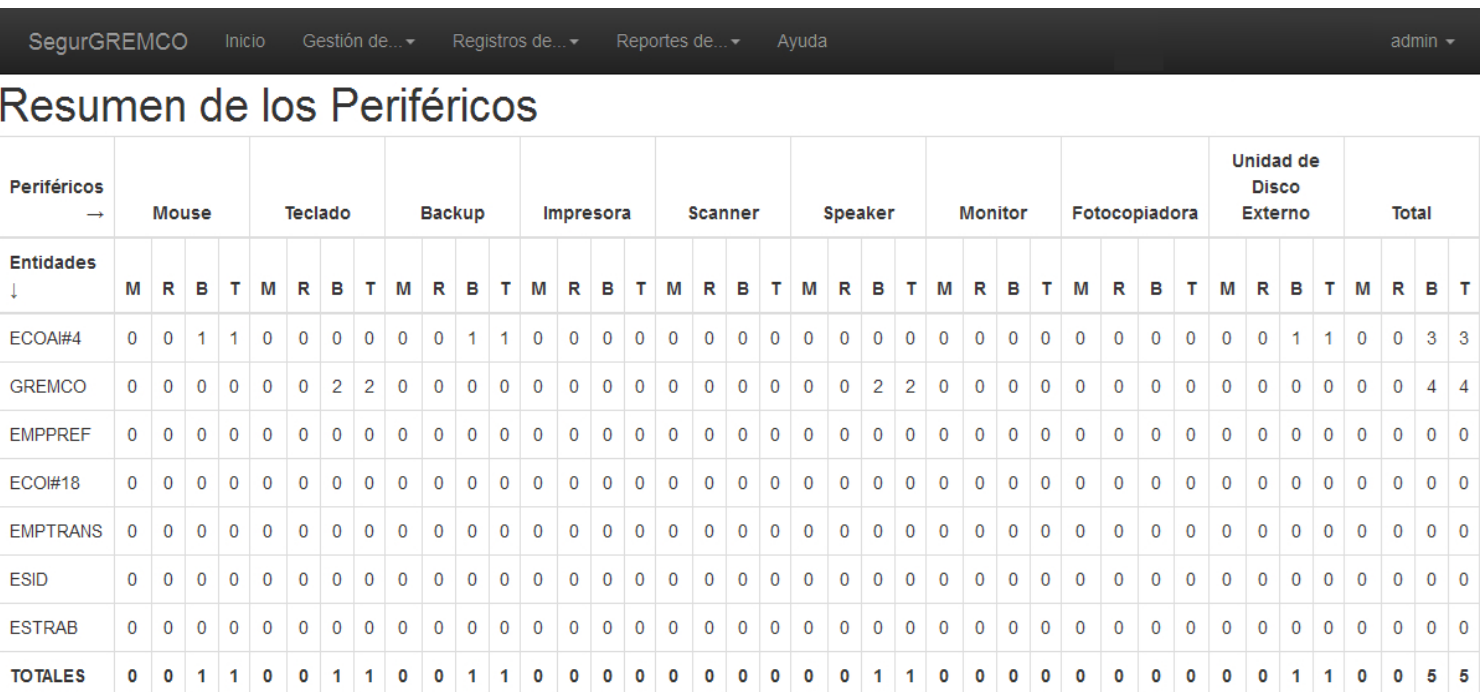

Figura 3. Reporte emitido por la aplicación web sobre los periféricos por empresa. 
La aplicación web brinda la facilidad de realizar salva(s) de seguridad de la base de datos cuando se estime necesario para garantizar la seguridad de la información ante la rotura del servidor. Se recomendó al cliente que las realizara periódicamente, las cuales podrían ser al finalizar cada jornada de trabajo y en diferentes fuentes de almacenamiento.

\section{DISCUSIÓN}

El éxito de un software se puede definir como calidad del sistema, de los datos y del servicio. La aplicación web impacta de manera positiva en los procesos y decisiones de GREMCO, al repercutir en todos estos aspectos. Con su implantación, no es necesario mejorar el equipamiento del que se dispone pues se desarrolló en función de sus requerimientos técnicos. Las tecnologías que requiere para su funcionamiento están basadas en software libre por lo que puede ser modificado en caso de cualquier error técnico. Se logra el ahorro de una serie de recursos como el papel al estar almacenada la información en formato digital. Representa beneficios en cuanto al factor tiempo, los errores humanos se minimizan y el control es más sistemático.

No genera empleo ni desempleo, se facilita en gran medida la realización de las actividades laborales. Se requiere de un mínimo de esfuerzo para utilizar la aplicación web, evita el estrés y mejora la calidad de vida al poder realizarse las operaciones con mayor rapidez. Se contribuye a elevar el nivel profesional y cultural de todos los trabajadores, ya que están interactuando con la tecnología que es utilizada en la mayor parte del mundo en cuanto a medios de comunicación e informatización.

El usuario, una vez que interactuó con la aplicación web fue capaz de percibir los beneficios que esta le proporciona para evaluar su calidad se empleó el criterio de expertos mediante el método Delphi. Éste consiste en la utilización sistemática del juicio intuitivo de un grupo de expertos para obtener un consenso de opiniones informadas. Es considerado como uno de los métodos subjetivos de pronosticación más confiables (Blanco, López y Mengual, 2010; García y Suárez, 2013).

Los expertos que evaluaron la calidad de la aplicación web fueron 31. Respondieron una encuesta que se confeccionó a partir de los siguientes indicadores generales de evaluación:

1. Resulta una interfaz amigable y fácil de operar.

2. Garantiza la disponibilidad de la información actual e histórica.

3. Facilita el análisis de la información actualizada.

4. Garantiza la seguridad de la información.

5. Constituye una herramienta útil para la toma de decisiones.

6. Disminuye el tiempo de respuesta.

7. Eleva la calidad de la información. 
8. Garantiza la obtención de todos los reportes que necesita.

9. Garantiza la obtención correcta de la información.

Después de realizado el procesamiento de la encuesta, se obvió que los aspectos $3,5,6,7,8$ y 9 son muy relevantes mientras que los aspectos 1,2 y 4 bastante relevante. Por tanto, estos resultados son un argumento a favor de la calidad y el impacto que tuvo la puesta en práctica en GREMCO de la aplicación web para la gestión de información de los servicios y medios de cómputo de los que disponen.

\section{CONCLUSIONES}

Con el desarrollo de la aplicación web propuesta en esta investigación GREMCO se cuenta con una solución informática que mejora la gestión y procesamiento de la información relacionada con los servicios y medios de cómputo de forma rápida y sencilla. Mediante su empleo se obtiene información en el momento que se necesite de manera detallada, precisa y con mayor consistencia, lo que mejora el análisis, la comprensión y eleva el nivel de eficiencia desde el punto de vista organizativo, de control y analítico. Avalan su calidad las pruebas de software y el método Delphi que se le aplicó. 


\section{REFERENCIAS BIBLIOGRÁFICAS}

Aladro, E. (2011). La teoría de la información ante las nuevas tecnologías de la comunicación. CIC : Cuadernos de Información y Comunicación, 16, 83-93.

Bagarotti, Y.C., Meneses, A. y Arias, Y. (2013). Experiencias durante la gestión de la calidad en proyectos que usan metodologías ágiles. Revista Ingeniería UC, 20(3), 45-53.

Blanco, J.E., López, A. y Mengual, S. (2010). Validación mediante método Delphi de un cuestionario para conocer las experiencias e interés hacia las actividades acuáticas con especial atención al Windsurf. ÁGORA, 12(1), 75-96.

Challenger, I., Díaz, Y. y Becerra, R.A. (2014). El lenguaje de programación Python. Ciencias Holguín, 20(2), 1-13.

Díaz, J. (2009). Las metodologías ágiles como garantía de calidad del software. Revista Española de Innovación, Calidad e Ingeniería del Software (REICIS), 5(3), 40-43.

Escobar, D.P. y Gualteros, A.C. (2013). Estudio de metodologías ágiles para proyectos de software en corto tiempo. Revista Tia, 1(2), 148-160.

Ferrada, X.V. y Serpell, A. (2009). La gestión del conocimiento y la industria de la construcción. Revista de la Construcción, 8(1), 46-58.

García, M. y Suárez, M. (2013). El método Delphi para la consulta a expertos en la investigación científica. Revista Cubana de Salud Pública, 39(2), 253-267.

Gauchat, J. (2012). El gran libro de HTML5, CSS3 y Javascript. Barcelona España.

Gutiérrez, C. (2008). Diseño web y arquitectura de información para sitios 2.0. Cuadernos de Información, 22, 58-65.

Hernández, R.L. y Greguas, D. (2010). Estándares de Diseño Web. Ciencias de la Información, 41(2), 69-71.

Lessin, J. (2013). Qué es Bootstrap y cómo funciona en el diseño web. Consultado: 25 de febrero de 2015, a partir de: <http://jorgelessin.com/que-es-bootstrap-y-comofunciona-en-el-diseno-web/>.

MICONS. (2014). Modelo del profesional de la especialidad Construcción Civil. Consultado: 7 de noviembre de 2014, a partir de: <http://micons.netcons.com.cu/>.

Miranda, M., Valdés, O., Pérez, I., Portelles, R., Sánchez, R. (2016). Metodología para la implementación de la gestión automatizada de controles de seguridad informática. Revista Cubana de Ciencias Informáticas, 10(2), 14-26.

Montesino, R., Baluja, W. y Porvén, J. (2013). Gestión automatizada e integrada de controles de seguridad informática. Ingeniería Electrónica, Automática y Comunicaciones, 34(1), 40-58. 
Tinoco, O., Rosales, P. P. y Salas, J. (2010). Criterios de selección de metodologías de desarrollo de software. Industrial Data, 13(2), 70-74.

Torres Lebrato, L. (2015). La gestión de información y la gestión del conocimiento. Revista Archivo Médico de Camagüey, 19(2), 96-98.

Vidal, M. J. y Araña, A. B. (2012). Gestión de la información y el conocimiento. Educación Médica Superior, 26(3), 474-484. 